\title{
Lindelöf property and the iterated continuous function spaces
}

\author{
by
}

\author{
G. A. Sokolov (Tomsk)
}

\begin{abstract}
We give an example of a compact space $X$ whose iterated continuous function spaces $C_{\mathrm{p}}(X), C_{\mathrm{p}} C_{\mathrm{p}}(X), \ldots$ are Lindelöf, but $X$ is not a Corson compactum. This solves a problem of Gul'ko (Problem 1052 in [11]). We also provide a theorem concerning the Lindelöf property in the function spaces $C_{\mathrm{p}}(X)$ on compact scattered spaces with the $\omega_{1}$ th derived set empty, improving some earlier results of Pol [12] in this direction.
\end{abstract}

1. Notation and terminology. Our terminology follows Arkhangel'skiı [1]. Given a topological space $X$, we denote by $C_{\mathrm{p}}(X)$ the space of real-valued continuous functions, equipped with the topology of pointwise convergence, and $C_{\mathrm{p}} C_{\mathrm{p}}(X), C_{\mathrm{p}} C_{\mathrm{p}} C_{\mathrm{p}}(X), \ldots$ are the iterated continuous function spaces.

We denote by $\mathcal{D}$ the discrete two-point space $\{0,1\}$ and $C_{\mathrm{p}}(X, \mathcal{D})=$ $\left\{f \in C_{\mathrm{p}}(X): f(X) \subset \mathcal{D}\right\}$.

We denote by $\omega_{1}$ the set of all countable ordinals.

A set $A \subset \omega_{1}$ is stationary if it intersects each closed set (in the order topology), unbounded in $\omega_{1}$; we call $A$ bistationary if both $A$ and $\omega_{1} \backslash A$ are stationary. For information concerning stationary sets needed in this paper we refer the reader to Jech [7], or Fleissner [3].

A topological space $X$ is $\aleph_{0}$-monolithic if for any countable subset $A \subset X$ the closure $\operatorname{cl}(A)$ has a countable network (see [1; Ch. II, $\S 6]$ ); a network for a space $Y$ is a family of sets such that each open set is the union of some subfamily of this family.

A topological space $X$ has countable tightness if for any $x \operatorname{in} \operatorname{cl}(A)$ there is an at most countable subset $B \subset A$ with $x \in \operatorname{cl}(B)$ (see [1]).

A compact space $X$ is a Corson compactum if $X$ can be embedded in the subspace of the Tikhonov product $\mathbb{R}^{\Gamma}$ of the real line consisting of functions vanishing at all but countably many points in $\Gamma$ (see [1], [9]). 
2. Main results. Gul'ko proved in [5] (a more detailed exposition in [6]) that for any weakly compact set $X$ in a Banach space (i.e. for any Eberlein compactum [1], [9]) the iterated function spaces $C_{\mathrm{p}}(X), C_{\mathrm{p}} C_{\mathrm{p}}(X), \ldots$ are Lindelöf. The author extended this result in [16] to the class of Corson compacta (essentially wider than the class of Eberlein compacta, see [1], [9]). Subsequently, Gul'ko conjectured that the Lindelöf property of all iterated continuous function spaces actually characterizes the class of Corson compacta (Problem 1052 in [11]).

We disprove this conjecture.

2.1. TheOrem. There exists a compact space $X$ (with the third derived set empty) such that all iterated continuous function spaces $C_{\mathrm{p}}(X)$, $C_{\mathrm{p}} C_{\mathrm{p}}(X), \ldots$ are Lindelöf, but $X$ is not a Corson compactum.

Our space $X$ is a compactum associated in a standard way with some special "ladder system" on the countable ordinals (see Section 4 for details). Similar function spaces were investigated earlier by Pol [13] and Ciesielski and Pol [2].

More precisely, we consider a class $\mathcal{S}$ of Lindelöf spaces, stable under the $C_{\mathrm{p}}$-operation, and we characterize those ladder systems on the countable ordinals which provide compacta $X$ with $X \in \mathcal{S}$.

In the definition of $\mathcal{S}$ we follow the author's paper [16], where more general classes of spaces (extending some classes defined by Gul'ko in [5], [6]) were considered. We discuss the class $\mathcal{S}$ in Section 3.

2.2. Remark. Some closely related questions concerning the iterated function spaces $C_{\mathrm{p}}(X), C_{\mathrm{p}} C_{\mathrm{p}}(X), \ldots$ were investigated by Sipachova [15] and Okunev [10]. In particular, Sipachova proved that if $X$ is an Eberlein compactum, then each of $C_{\mathrm{p}}(X), C_{\mathrm{p}} C_{\mathrm{p}}(X), \ldots$ is a Lindelöf $\Sigma$-space ( $\equiv$ countably determined space, see $[9]$ ), and Okunev extended this result to the class of Gul'ko compacta (see definition in [9]). The result of Okunev is definitive in this direction: Gul'ko compacta $X$ are characterized by the property that $C_{\mathrm{p}}(X)$ is a Lindelöf $\Sigma$-space.

Our next result provides information about the Lindelöf property in the function spaces $C_{\mathrm{p}}(X)$ on compacta whose $\omega_{1}$ th derived set is empty. Some results in this direction were obtained by Pol [12].

Before stating the result let us recall that, given a topological space $E$, the $G_{\delta}$-modification of $E$ is the set $E$ equipped with the topology generated by all countable intersections of open sets in $E$.

2.3. THEOREM. Let $X$ be a compact scattered space with the $\omega_{1}$ th derived set empty. Then the following properties are equivalent.

(a) $C_{\mathrm{p}}(X)$ is Lindelöf,

(b) the $G_{\delta}$-modification of $C_{\mathrm{p}}(X, \mathcal{D})$ is Lindelöf, 
(c) $X$ is $\aleph_{0}$-monolithic.

Let us recall that $X$ being scattered, the weak topology on the function space coincides on norm-bounded sets with the pointwise topology (see [14]). Hence, in Theorem 2.3 we could as well consider the Banach function space $C(X)$ equipped with the weak topology.

2.4. Remark. Assuming Martin's Axiom and the negation of the Continuum Hypothesis, the implication $(\mathrm{a}) \Rightarrow(\mathrm{c})$ was proved by Reznichenko for arbitrary compact Hausdorff spaces (see [17], [1; Ch. IV, §8]).

3. A stable class $\mathcal{S}$ of Lindelöf spaces. In [16], for any infinite cardinal $\tau$, the author introduced two classes of topological spaces, $\mathcal{D}(\tau)$ and $\mathcal{L}(\tau)$, which are in duality with respect to the $C_{\mathrm{p}}$-operation. The classes $\mathcal{D}(\tau)$ and $\mathcal{L}(\tau)$ extended some earlier constructions of Gul'ko [5], [6], and the ideas developed by Gul'ko in these papers were basic in investigation of properties of the classes.

The class $\mathcal{S}$ we consider is contained in the intersection $\mathcal{D}\left(\aleph_{0}\right) \cap \mathcal{L}\left(\aleph_{0}\right)$. The properties of $\mathcal{S}$ we need could be derived from the general results in [16]. However, in the case of $\mathcal{S}$, the proofs can be made more direct and clear, and we decided to include them for the reader's convenience.

3.1. Definition. The class $\mathcal{S}$ consists of all topological spaces $X$ satisfying the following two conditions:

(a) for every $n \in \mathbb{N}$, the space $X^{n}$ is Lindelöf and has countable tightness,

(b) if $F_{n} \subset X^{n}$ for $n=1,2, \ldots$ is a sequence of closed subsets, then there exists a mapping $r: X \rightarrow X$ such that $r(X)$ has a countable network and $(r \times \ldots \times r)\left(F_{n}\right) \subset F_{n}$ for every $n \in \mathbb{N}$.

\subsection{Theorem. If $X \in \mathcal{S}$ then $C_{\mathrm{p}}(X) \in \mathcal{S}$.}

Proof. Let $X_{m}=X \oplus \ldots \oplus X$ be the discrete union of $m$ copies of $X$.

Claim 1. If $F_{n m} \subset\left(X_{m}\right)^{n}$ are closed sets, $n, m \in \mathbb{N}$, then there exists a mapping $r: X \rightarrow X$ such that $r(X)$ has a countable network and $\left(r_{m} \times \ldots\right.$ $\left.\ldots \times r_{m}\right)\left(F_{n m}\right) \subset F_{n m}$, where the $r_{m}: X_{m} \rightarrow X_{m}$ are induced by $r$.

Indeed, consider $\left(X_{m}\right)^{n}$ as a finite discrete union of clopen subspaces $X_{n m k}$, each homeomorphic to $X^{n}$, and set $F_{n m k}=F_{n m} \cap X_{n m k}$. Let $\pi$ : $\mathbb{N} \times \mathbb{N} \times \mathbb{N} \rightarrow \mathbb{N}$ be a bijection such that $\pi(n, m, k) \geq n$ for every $n, m, k$ in $\mathbb{N}$. Then we can consider each $F_{n m k}$ as a closed subset of $X^{\pi(n, m, k)}$. Now Claim 1 follows from condition (b) of Definition 3.1.

For any $x=\left(x_{1}, \ldots, x_{n}\right) \in X^{n}$ and an $n$-tuple $I=\left(I_{1}, \ldots, I_{n}\right)$ of open intervals in the real line with rational ends, let

$$
W(x, I)=\left\{f \in C_{\mathrm{p}}(X): f\left(x_{i}\right) \in I_{i} \text { for } i=1, \ldots, n\right\} .
$$


If $f \in C_{\mathrm{p}}(X), x \in \operatorname{cl}(A)$ and $f\left(x_{i}\right) \in I_{i}$ for every $i$ then there exists $y \in A$ with $f\left(y_{i}\right) \in I_{i}$ for all $i$ and therefore we have

Claim 2. Let $n \in \mathbb{N}, A \subset X^{n}$ and I be a fixed n-tuple as above. Then $W(x, I) \subset \bigcup\{W(y, I): y \in A\}$ for any $x \in \operatorname{cl}(A)$.

Given $n \in \mathbb{N}$, denote by $\mathcal{B}_{n}$ the countable family of all $n$-tuples $I=$ $\left(I_{1}, \ldots, I_{n}\right)$ of open intervals in the real line with rational ends.

We pass to the proof that $C_{\mathrm{p}}(X)$ meets the conditions of Definition 3.1.

Let $H_{m} \subset\left(C_{\mathrm{p}}(X)\right)^{m}$ be a sequence of closed subsets. Observe that $\left(C_{\mathrm{p}}(X)\right)^{m}$ can be identified with $C_{\mathrm{p}}\left(X_{m}\right)$, where $X_{m}$ was defined at the beginning of the proof. For any $n, m$ in $\mathbb{N}$ and $I \in \mathcal{B}_{n}$ denote by $\mathcal{U}_{n m}(I)$ the family of all sets $W(x, I)$ with $x \in\left(X_{m}\right)^{n}$, disjoint from $H_{n}$. Let

$$
F_{n m}(I)=\left\{x \in\left(X_{m}\right)^{n}: W(x, I) \in \mathcal{U}_{n m}(I)\right\} .
$$

By Claim 2, each $F_{n m}(I)$ is closed in $\left(X_{m}\right)^{n}$. Therefore Claim 1 provides us with a mapping $r: X \rightarrow X$ such that $r(X)$ has a countable network and $\left(r_{m} \times \ldots \times r_{m}\right)\left(F_{n m}(I)\right) \subset F_{n m}(I)$ for any $n, m$ in $\mathbb{N}$ and $I \in \mathcal{B}_{n}$.

Let $r^{*}: C_{\mathrm{p}}(X) \rightarrow C_{\mathrm{p}}(X)$ be the dual mapping $f \rightarrow r^{*}(f)=f \circ r$, for $f \in C_{\mathrm{p}}(X)$. Notice that $r^{*} \times \ldots \times r^{*}=r_{m}^{*}$.

We show that $r_{m}^{*}\left(H_{m}\right) \subset H_{m}$ for every $m \in \mathbb{N}$. Suppose on the contrary that there exists $f \in H_{m}$ with $r_{m}^{*}(f) \notin H_{m}$. Then there exists an open set $U$ in $C_{\mathrm{p}}\left(X_{m}\right)$ containing $r_{m}^{*}(f)$ and disjoint from $H_{m}$. We can assume that $U=W(x, I)$ for some $n \in \mathbb{N}, x \in\left(X_{m}\right)^{n}$ and $I \in \mathcal{B}_{n}$. Since $W(x, I) \cap H_{m}$ $=\emptyset$, we have $x \in F_{n m}(I)$, and therefore $\left(r_{m} \times \ldots \times r_{m}\right)(x) \in F_{n m}(I)$. It follows that $W\left(\left(r_{m} \times \ldots \times r_{m}\right)(x), I\right) \cap H_{m}=\emptyset$. But $r_{m}^{*}(f) \in W(x, I)$ if, and only if, $f \in W\left(\left(r_{m} \times \ldots \times r_{m}\right)(x), I\right)$. Therefore $f \notin H_{m}$, contradicting our choice of $f$. This proves that $r_{m}^{*}\left(H_{m}\right) \subset H_{m}$.

Since $C_{\mathrm{p}}\left(r_{m}(X)\right)$ has a countable network (because $r_{m}(X)$ has one, see [1]) and we can identify $r_{m}^{*}\left(C_{\mathrm{p}}(X)\right)$ with $C_{\mathrm{p}}\left(r_{m}(X)\right)$, we have verified condition (b) of Definition 3.1 for $C_{\mathrm{p}}(X)$.

We now check (a), i.e. we prove that each finite product $\left(C_{\mathrm{p}}(X)\right)^{m}=$ $C_{\mathrm{p}}\left(X_{m}\right)$ is Lindelöf.

Fix $m \in \mathbb{N}$, and let $\mathcal{U}$ be an open cover of $C_{\mathrm{p}}\left(X_{m}\right)$ consisting of basic neighbourhoods $W(x, I)$ defined above. For each $n$, and $I \in \mathcal{B}_{n}$, let

$$
\begin{aligned}
& \mathcal{U}_{n}(I)=\left\{W(x, I): x \in\left(X_{m}\right)^{n} \text { and } W(x, I) \in \mathcal{U}\right\}, \\
& A_{n}(I)=\left\{x \in\left(X_{m}\right)^{n}: W(x, I) \in \mathcal{U}_{n}(I)\right\} .
\end{aligned}
$$

Applying Claim 1 to $\operatorname{cl}\left(A_{n}(I)\right)$ we get a mapping $r: X \rightarrow X$ whose image $r(X)$ has a countable network and $S_{n}(I)=\left(r_{m} \times \ldots \times r_{m}\right)\left(\operatorname{cl}\left(A_{n}(I)\right)\right) \subset$ $\operatorname{cl}\left(A_{n}(I)\right)$ for each $n$. The sets $S_{n}(I)$ have countable networks, and therefore there are countable sets $B_{n}(I)$ dense in $S_{n}(I)$. Since $\left(X_{m}\right)^{n}$ has countable tightness, each $x \in B_{n}(I)$ is in the closure of a countable subset of $A_{n}(I)$, 
and therefore, by Claim 2, each $W(x, I)$ with $x \in B_{n}(I)$ is covered by a countable subfamily of $\mathcal{U}_{n}(I)$.

Therefore, to complete the proof we verify that the countable collection

$$
\mathcal{W}=\left\{W(x, I): x \in B_{n}(I), n \in \mathbb{N}, I \in B_{n}\right\}
$$

covers $C_{\mathrm{p}}\left(X_{m}\right)$.

Let $f \in C_{\mathrm{p}}\left(X_{m}\right)$. There exists $W(x, I) \in \mathcal{U}$ with $r_{m}^{*}(f) \in W(x, I)$, i.e. $f \in W\left(\left(r_{m} \times \ldots \times r_{m}\right)(x), I\right)$. There exists $n$ such that $x \in A_{n}(I)$ and then $\left(r_{m} \times \ldots \times r_{m}\right)(x) \in S_{n}(I)$. Since $B_{n}(I)$ is dense in $S_{n}(I)$, applying Claim 2 again, we conclude that $W\left(\left(r_{m} \times \ldots \times r_{m}\right)(x), I\right)$ is covered by elements $W(y, I)$ with $y \in B_{n}(I)$, and therefore $f$ is in the union of the family $\mathcal{W}$.

Finally, the countable tightness of the finite powers $\left(C_{\mathrm{p}}\left(X_{m}\right)\right)^{n}$ follows from the Arkhangel'skiı̌-Pytkeev theorem [1; Ch. II, §1], since all finite powers $\left(X_{m}\right)^{n}$ are Lindelöf.

3.3. Remark. It was proved in [16] that all Corson compacta are in $\mathcal{S}$. In particular, all iterated function spaces $C_{\mathrm{p}}(X), C_{\mathrm{p}} C_{\mathrm{p}}(X), \ldots$ for $X$ a Corson compactum are Lindelöf.

This is closely related to the well-known results of Gul'ko [1], [4-6] concerning retraction systems on Corson compacta.

4. The compacta $X_{A}$ associated with ladder systems on $\omega_{1}$ and the proof of Theorem 2.1. Given a countable limit ordinal $\alpha$, a ladder on $\alpha$ is a set $S_{\alpha}=\{\alpha(1), \alpha(2), \ldots\}$ of isolated ordinals from $\alpha$ such that $\alpha(1)<\alpha(2)<\ldots$ and $\alpha=\sup _{i} \alpha(i)$. Let $A$ be a set of countable limit ordinals. An $A$-ladder system on $\omega_{1}$ is a collection $\left\langle S_{\alpha}: \alpha \in A\right\rangle$, where $S_{\alpha}$ is a ladder on $\alpha$. With each $A$-ladder system on $\omega_{1}$ we associate a compact space $X_{A}$ in the following standard way: we give the set $\omega_{1}$ a locally compact topology by making the points in $\omega_{1} \backslash A$ isolated and taking as a base of neighbourhoods of a point $\alpha \in A$ the sets $\{\alpha\} \cup\left(S_{\alpha} \backslash F\right)$, where $F$ is a finite set, and we let $X_{A}$ be the one-point compactification of this space, $\omega_{1}$ being the point at infinity.

4.1. Proposition. $X_{A}$ is a Corson compactum if and only if $A$ is a nonstationary set in $\omega_{1}$.

Proof. Suppose $A$ is a nonstationary set in $\omega_{1}$. Then there exists a closed (in the order topology) unbounded set $B$ in $\omega_{1}$ with $A \cap B=\emptyset$. Without loss of generality we may assume that $1 \in B$. Then for every $\alpha \in \omega_{1}$ there exists $\beta(\alpha) \in B$ such that $\beta(\alpha) \leq \alpha<\beta(\alpha)^{+}$, where $\beta(\alpha)^{+}$is the ordinal in $B$ next to $\beta(\alpha)$.

Let $\alpha \in A$ and $S_{\alpha}$ be a ladder on $\alpha$. For any $m \in \mathbb{N}$ set $S_{\alpha}^{m}=\{\alpha(n)$ : $n \geq m\}$. Define

$$
\mathcal{U}=\{\{\alpha\}: \alpha \notin A\} \cup\left\{\left(\beta(\alpha), \beta(\alpha)^{+}\right) \cap S_{\alpha}^{m}: \alpha \in A, m \in \mathbb{N}\right\} .
$$


It is easy to check that $\mathcal{U}$ is a point-countable family of clopen sets such that for any pair of distinct points in $X_{A}$ there is a set in $\mathcal{U}$ containing exactly one of them. By a Rosenthal-type characterization theorem [1], [9] we conclude that $X_{A}$ is a Corson compactum.

Now let $A$ be a stationary set in $\omega_{1}$. Assume on the contrary that $X_{A}$ is a Corson compactum and therefore it has a point-countable family $\mathcal{U}$ consisting of open $F_{\sigma}$-sets such that given two points in $X$, some element in $\mathcal{U}$ contains exactly one of them. For every $\alpha \in A$ fix $U_{\alpha} \in \mathcal{U}$ such that $\alpha \in U_{\alpha}$. We may assume that the sets $U_{\alpha}$ are distinct for distinct ordinals $\alpha$. For each $\alpha \in A$ there exists $h(\alpha) \in U_{\alpha}$ such that $h(\alpha)<\alpha$, since $\alpha$ is nonisolated in $X_{A}$. By the pressing-down lemma we have $h(\alpha)=\alpha_{0}$ for all $\alpha$ in a stationary subset $A^{\prime}$ of $A$. The point $\alpha_{0}$ belongs to every $U_{\alpha}$ with $\alpha \in A^{\prime}$, hence $\mathcal{U}$ is not point-countable.

4.2. Proposition. $X_{A}$ is in the class $\mathcal{S}$ if and only if $\omega_{1} \backslash A$ is a stationary set in $\omega_{1}$.

Proof. Since $X_{A}$ is a compactum with countable tightness each finite power of $X_{A}$ has countable tightness (by Malykhin's theorem [8]) and the Lindelöf property. Hence we need only verify condition (b) of Definition 3.1 for $X_{A}$.

Let $\omega_{1} \backslash A$ be a stationary set in $\omega_{1}$ and $F_{n} \subset\left(X_{A}\right)^{n}$ be a sequence of closed subsets.

Let $L\left(\omega_{1}\right)$ be the set $\omega_{1} \cup\left\{\omega_{1}\right\}$ with the topology where all points in $\omega_{1}$ are isolated and the neighbourhoods of $\omega_{1}$ contain all but countably many points in $L\left(\omega_{1}\right)$. The space $L\left(\omega_{1}\right)$ is the $G_{\delta}$-modification of $X_{A}$, i.e. open sets in $L\left(\omega_{1}\right)$ are countable intersections of open sets in $X_{A}$. In particular, each $F_{n}$ is closed in $L\left(\omega_{1}\right)^{n}$.

The mapping $r_{\beta}: L\left(\omega_{1}\right) \rightarrow L\left(\omega_{1}\right)$ defined by

$$
r_{\beta}(\alpha)= \begin{cases}\alpha & \text { if } \alpha<\beta, \\ \omega_{1} & \text { otherwise }\end{cases}
$$

is a continuous retraction. By Gul'ko's theorem [1; IV.3.12] the set

$$
C_{n}=\left\{\beta \in \omega_{1}:\left(r_{\beta} \times \ldots \times r_{\beta}\right)\left(F_{n}\right) \subset F_{n}\right\}
$$

is closed and unbounded in $\omega_{1}$ for every $n \in \mathbb{N}$. Hence there exists $\beta \in$ $\left(\bigcap_{n} C_{n}\right) \backslash A, \omega_{1} \backslash A$ being stationary. Notice that the same $r_{\beta}$ considered as a mapping from $X_{A}$ into itself is continuous if, and only if, $\beta \notin A$. Furthermore, the image of $r_{\beta}$ is countable, so $r=r_{\beta}$ satisfies 3.1(b). This proves the "if" part of the proposition.

To check the reverse implication, assume that there exists a closed unbounded set $C$ in $\omega_{1}$ contained in $A$. Without loss of generality we can assume that $1 \in C$. 
For any $\alpha$, let $\alpha^{+}$be the successor of $\alpha$ in $C$ and let $\beta_{1}(\alpha), \beta_{2}(\alpha), \ldots$ enumerate the points in the interval $\left[\alpha, \alpha^{+}\right)$.

Define a sequence of closed subsets of $X^{2}$ by

$$
F_{0}=\{(1,1)\} \quad \text { and } \quad F_{n}=\operatorname{cl}\left(\left\{\left(\alpha, \beta_{n}(\alpha)\right): \alpha \in C\right\}\right), \quad \text { for } n \in \mathbb{N} .
$$

Since $C \subset A$, every infinite sequence in $C$ converges in $X_{A}$ to $\omega_{1}$. It follows that $F_{n} \cap\left(\{\alpha\} \times X_{A}\right)=\left\{\left(\alpha, \beta_{n}(\alpha)\right)\right\}$ for $\alpha \in C$.

Assume that $r: X_{A} \rightarrow X_{A}$ is continuous and $(r \times r)\left(F_{n}\right) \subset F_{n}$ for every $n=1,2, \ldots$ We show that $r$ is the identity map. We have $r(1)=1$ because $(r \times r)\left(F_{0}\right) \subset F_{0}$. Suppose that $r(\gamma)=\gamma$ for all $\gamma<\beta$. If $\beta \in C$ then $\beta$ is an accumulation point of $[1, \beta)$, and therefore $r(\beta)=\beta$. Let $\beta \notin C$. Then $\beta=\beta_{n}(\alpha)$ for some $\alpha \in C$ and $n \in \mathbb{N}$. Observe that $\alpha<\beta$ and $r(\alpha)=\alpha$. Hence $(r \times r)\left(F_{n}\right) \subset F_{n}$ implies $r\left(\beta_{n}(\alpha)\right)=\beta_{n}(\alpha)$. This completes the inductive proof of the "only if" part of the proposition.

4.3. Corollary. If $A$ is a bistationary set in $\omega_{1}$ then all iterated function spaces $C_{\mathrm{p}}\left(X_{A}\right), C_{\mathrm{p}} C_{\mathrm{p}}\left(X_{A}\right), \ldots$ are Lindelöf, but $X_{A}$ is not a Corson compactum.

5. Proof of Theorem 2.3. For any $Y$ closed in $X$ the restriction operator $R(f)=\left.f\right|_{Y}$ maps continuously $C_{\mathrm{p}}(X)$ onto $C_{\mathrm{p}}(Y)$; in particular, if $C_{\mathrm{p}}(X)$ is Lindelöf, so is $C_{\mathrm{p}}(Y)$. By a result of Pol [12], we infer that if $C_{\mathrm{p}}(X)$ is Lindelöf and $Y$ is separable, then $Y$ is metrizable. This gives the implication $(\mathrm{a}) \Rightarrow(\mathrm{c})$.

The implication $(b) \Rightarrow(a)$ was proved in [13].

To show $(\mathrm{c}) \Rightarrow(\mathrm{b})$, let $X$ be a compact $\aleph_{0}$-monolithic scattered space. Denote by $X^{(\alpha)}$ the $\alpha$ th derived set of $X$ (see [14]). Since $X^{\left(\omega_{1}\right)}=\emptyset$, there is a countable ordinal $\alpha$ such that $X^{(\alpha)}$ is finite; we then call $\alpha$ the height of $X$. We can restrict ourselves to the case where $X^{(\alpha)}$ is a singleton $\left\{x^{*}\right\}$. We can also concentrate on the space

$$
C_{\mathrm{p}}^{0}(X, \mathcal{D})=\left\{f \in C_{\mathrm{p}}(X, \mathcal{D}): f\left(x^{*}\right)=0\right\}
$$

instead of $C_{\mathrm{p}}(X, \mathcal{D})$, the latter being the union of two disjoint closed copies of the former.

Now observe that a base of the $G_{\delta}$-modification of $C_{\mathrm{p}}^{0}(X, \mathcal{D})$ consists of the sets

$$
U(A, \varphi)=\left\{f \in C^{0}(X, \mathcal{D}):\left.f\right|_{A}=\varphi\right\},
$$

where $A$ is a countable subset of $X$ and $\varphi: A \rightarrow \mathcal{D}$ is a function. We prove that $C^{0}(X, \mathcal{D})$ is a Lindelöf space in the $G_{\delta}$-topology by induction on the height of $X$.

The case $\alpha=0$ is evident. 
Let $\alpha$ be a limit ordinal. Then there exists a sequence $\alpha_{n}<\alpha$ converging to $\alpha$, and we have $C^{0}(X, \mathcal{D})=\bigcup_{n} E\left(\alpha_{n}\right)$, where

$$
E\left(\alpha_{n}\right)=\left\{f \in C^{0}(X, \mathcal{D}):\left.f\right|_{X^{(\alpha)}} \equiv 0\right\} .
$$

Let $Z_{n}$ be the factor space obtained by collapsing the set $X^{(\alpha)}$ to a point $z_{n}^{*}$. Clearly, $E\left(\alpha_{n}\right)$ is homeomorphic to

$$
C^{0}\left(Z_{n}, \mathcal{D}\right)=\left\{f \in C^{0}(Z, \mathcal{D}): f\left(z_{n}^{*}\right)=0\right\} .
$$

Furthermore, $Z_{n}^{(\alpha)}=\emptyset$ and $Z_{n}$ is $\aleph_{0}$-monolithic as a continuous image of the $\aleph_{0}$-monolithic space $X$. By the inductive assumption, each $E\left(\alpha_{n}\right)$, and therefore $C_{\mathrm{p}}^{0}(X, \mathcal{D})$, is Lindelöf.

Let $\alpha=\beta+1$. The derived set $X^{(\beta)}$ is homeomorphic to the one-point compactification of some discrete space $\Gamma$. Write $X^{(\beta)}=\left\{x^{*}\right\} \cup\left\{x_{\gamma}: \gamma \in \Gamma\right\}$ and let

$$
\begin{aligned}
& C_{n}=\left\{f \in C^{0}(X, \mathcal{D}):\left|\left\{\gamma \in \Gamma: f\left(x_{\gamma}\right)=1\right\}\right| \leq n\right\}, \quad n=1,2, \ldots, \\
& E_{\gamma}=\left\{f \in C_{1}: f\left(x_{\gamma}\right)=1\right\} .
\end{aligned}
$$

Obviously, $C^{0}(X, \mathcal{D})=\bigcup_{n} C_{n}$, hence it is sufficient to prove that $C_{n}$ is Lindelöf for every $n$. The case $n=0$ is similar to the case just considered and by the inductive assumption $E_{\gamma}$ is Lindelöf for every $\gamma \in \Gamma$. Next, note that $C_{n}$ is the continuous image of $\left(C_{1}\right)^{n}$ under the mapping $\left(f_{1}, \ldots, f_{n}\right) \rightarrow$ $\max \left(f_{1}, \ldots, f_{n}\right)$. So it remains to prove that $C_{1}$ is a Lindelöf space in the $G_{\delta}$-topology.

Let $\mathcal{U}$ be an open cover of $C_{1}$. We have already noticed that the subspace $C_{0}$ of $C_{1}$ is Lindelöf; let $\mathcal{U}^{\prime}=\left\{V_{n}: n \in \mathbb{N}\right\}$ be a subfamily of $\mathcal{U}$ covering $C_{0}$. We may assume that $V_{n}=U\left(A_{n}, \varphi_{n}\right)$ for $n \in \mathbb{N}$. As every $E_{\gamma}$ is Lindelöf for $\gamma \in \Gamma$ we need only prove that $\bigcup \mathcal{U}^{\prime}$ covers all $E_{\gamma}$ except possibly for a countable set. Suppose on the contrary that there are $f_{\gamma} \in E_{\gamma}$ with $f_{\gamma} \notin \bigcup_{n} V_{n}$ for $\gamma \in \Gamma^{\prime} \subset \Gamma, \Gamma^{\prime}$ being uncountable. Let $A=\bigcup_{n} A_{n}$ and let $\gamma \in \Gamma^{\prime}$ be such that $x_{\gamma} \notin \operatorname{cl}(A)$. Then there is a clopen neighbourhood $W$ of $x_{\gamma}$ such that $W \cap \operatorname{cl}(A)=\emptyset$. Let $g_{\gamma}=f_{\gamma} \chi_{X \backslash W}$, where $\chi_{X \backslash W}$ is the characteristic function of the set $X \backslash W$. Since $g_{\gamma} \in C_{0}$, we have $g_{\gamma} \in V_{n}$ for some $n$. But by the assumption $g_{\gamma}$ coincides with $f_{\gamma}$ on $A_{n}$ and hence $f_{\gamma} \in U\left(A_{n}, \varphi_{n}\right)=V_{n}$. This contradiction proves the claim.

6. Open problems. Is there a compact space $X$ such that $C_{\mathrm{p}}(X)$ is Lindelöf but $C_{\mathrm{p}} C_{\mathrm{p}}(X)$ is not? The same question can be posed for $X$ being scattered $\aleph_{0}$-monolithic.

The answer is unknown even for $X_{A}$ from Section 4 with $A$ an arbitrary set.

Gul'ko [4] proved that if $X$ is the $\Sigma$-product of the real lines then $C_{\mathrm{p}}(X)$ is Lindelöf. The uncountable $\Sigma$-product $X$ is not Lindelöf and can be em- 
bedded as a closed subspace in $C_{\mathrm{p}} C_{\mathrm{p}}(X)$. Therefore the compactness requirement in the above question is essential.

\section{References}

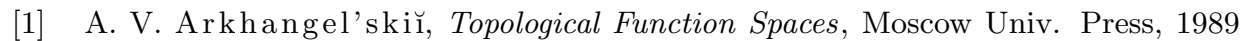
(in Russian); English transl.: Kluwer Acad. Publ., Dordrecht 1992.

[2] K. Ciesielski and R. Pol, A weakly Lindelöf function space $C(K)$ without any continuous injection into $c_{0}(\Gamma)$, Bull. Acad. Polon. Sci. 32 (1984), 681-688.

[3] W. G. Fleissner, Applications of stationary sets in topology, in: Surveys in General Topology, Academic Press, 1980, 163-193.

[4] S. P. Gul'ko, On properties of subsets of $\Sigma$-products, Dokl. Akad. Nauk SSSR 237 (1977), 505-508 (in Russian); English transl.: Soviet Math. Dokl. 18 (1977), $1438-1442$.

[5] - On properties of some function spaces, Dokl. Akad. Nauk SSSR 243 (1978), 839-842 (in Russian); English transl.: Soviet Math. Dokl. 19 (1978), 1420-1424.

[6] -, On properties of function spaces, in: Seminar on General Topology, Moscow Univ. Press, 1981, 8-41 (in Russian).

[7] T. Jech, Set Theory, Academic Press, New York 1978.

[8] V. I. Malykhin, On the tightness and the Suslin number of $\exp X$ and of a product of spaces, Dokl. Akad. Nauk SSSR 203 (1972), 1001-1003 (in Russian); English transl.: Soviet Math. Dokl. 13 (1972), 496-499.

[9] S. Negrepontis, Banach spaces and topology, in: Handbook of Set-Theoretic Topology, North-Holland, Amsterdam 1984, 1045-1142.

[10] O. G. Okunev, On the weak topology of conjugate spaces and the t-equivalence relation, Mat. Zametki 46 (1989), 53-59 (in Russian).

[11] Open Problems in Topology, J. van Mill and G. M. Reed (eds.), North-Holland, Amsterdam 1990.

[12] R. Pol, Concerning function spaces on separable compact spaces, Bull. Acad. Polon. Sci. 25 (1977), 993-997.

[13] -, A function space $C(X)$ which is weakly Lindelöf but not weakly compactly generated, Studia Math. 64 (1979), 279-284.

[14] Z. Semadeni, Banach Spaces of Continuous Functions, PWN, Warszawa 1971.

[15] O. V. Sipachova, The structure of iterated function spaces in the topology of pointwise convergence for Eberlein compacta, Mat. Zametki 47 (1990), 91-99 (in Russian).

[16] G. A. Sokolov, On Lindelöf spaces of continuous functions, ibid. 36 (1986), 887894 (in Russian).

[17] E. A. Reznichenko, Convex and compact subsets of function spaces and locally convex spaces, Ph.D. thesis, Moscow Univ., 1992 (in Russian).

FACULTY OF MECHANICS AND MATHEMATICS

TOMSK STATE UNIVERSITY

PR. LENINA 36

634010 TOMSK, RUSSIA 